\title{
Power System Optimization in Terms of Uncertainty of Initial Information
}

\author{
Tulkin Gayibov ${ }^{1, *}$, Bekzod Pulatov ${ }^{1}$, Sherxon Latipov ${ }^{1}$, and Gulnaz Turmanova ${ }^{2}$ \\ ${ }^{1}$ Tashkent State Technical University named after Islam Karimov, Uzbekistan \\ ${ }^{2}$ Karakalpak State University, Uzbekistan
}

\begin{abstract}
In the article the problem of power system optimization under partial uncertainty of initial information is considered. The possibilities of using the methods of determinization to solve the problem of power system optimization taking into account the regime and technological constraints in the form of equality and inequality are researched. In basis of performed calculation studies using the example of the test scheme of power system, it was found that the optimization algorithm by the method of determinization can be effectively used for this proposes.
\end{abstract}

\section{Introduction}

The problem of optimization of power system development and functioning is a difficult problem of nonlinear mathematical programming with many limiting conditions in the form of equality and inequality imposed on adjustable parameters and on some of their functions. These problems are also characterized, like similar problem for any large system, by the presence of various kinds of initial information. Currently, the initial data used to solve such problems can be divided into four types: deterministic, probabilistic, fuzzy, and interval.

The deterministic data gives a complete picture of the state of object, which meets the requirements of this problem. The last three types of initial data are uncertain data. With a probabilistic initial data, the laws of distribution of probabilistic quantities in known ranges of their possible values are usually set. Fuzzy initial data suggests their task by formulated linguistic distribution of possible parameter values. In the case of interval initial information, the intervals of possible values of the initial parameter (or state) are usually known, and no laws are known about their possible values (or states) in this interval.

Currently, the methods and algorithms for solving power system optimization problems in the conditions of deterministic initial data are sufficiently developed [1-7]. The solution of the problem in conditions of probabilistic initial data is carried out by probabilistic-statistical methods. They provide for the use of the mathematical expectation functions of the objective function and the constraint functions of probabilistic quantities. In this case, the calculation algorithms do not differ much from the algorithms for deterministic initial data. The optimization problems in the conditions of fuzzy initial data are solved by methods of the theory of fuzzy sets $[8,9]$. In this paper, we consider the problem of Power system optimization in the conditions of interval (partial) uncertainty of the initial parameter. In the existing literature, there are no an advanced algorithms for optimization of the development and functioning of power system, taking into account all the constraints in conditions of partial uncertainty of the initial information. In [10-12], the efficient algorithm of power system optimization in terms of partial uncertainty of initial information is described. The algorithm is based on the sequential solution of deterministic problem for the values of initial parameter selected in a given interval and select the best plan for various criteria. In this case, preference is given to the criteria of minimax and minmax risk. At the same time, in these works, the questions of taking into account of constraints in the form of inequality remains opened. The algorithms proposed in [13] for taking into account inequality constraints during optimization under the conditions of probability and partial uncertainty of the initial information do not completely overcome the noted difficulties. In some cases, the determination of a conditionally optimal plan, where all the specified constrains are satisfied for all the possible values of the initial parameter in a given range, requires additional complex calculations. In [14-16] an algorithm of optimization under conditions of interval uncertainty of the initial parameter by the method of determinization, taking into account the constraints is described. This paper presents the results of study of possibility of using this algorithm to solve problems of optimization of a development and functioning of power system.

\footnotetext{
* Corresponding author: tulgayibov@gmail.com
} 


\section{The optimization algorithm}

We consider the possibilities of using the algorithm of optimization in terms of uncertainty by determinization method for solving the problem of power system development optimization, which is formulated as follows: minimize the function of estimated costs

$$
3\left(a_{1}, a_{2}, \ldots, a_{m}, x_{1}, x_{2}, \ldots, x_{n}\right) \rightarrow \min
$$

taking into account

- simple constraints imposed on adjustable parameters

$$
x_{i}^{\min } \leq x_{i} \leq x_{i}^{\max }, \quad i=\overline{1, n} \text {; }
$$

- functional constraints in the form of equality and inequality imposed on some functions of adjustable parameters

$$
\begin{aligned}
& f_{j}\left(a_{1}, a_{2}, \ldots, a_{m}, x_{1}, x_{2}, \ldots, x_{n}\right)=\bar{f}_{j}, \quad j=\overline{1, N} ; \\
& g_{l}\left(a_{1}, a_{2}, \ldots, a_{m}, x_{1}, x_{2}, \ldots, x_{n}\right) \leq \bar{g}_{l}, \quad l=\overline{1, L},
\end{aligned}
$$

where $\bar{f}_{j}, \bar{g}_{l}$ are specified values of the constraint functions.

At partial uncertainty of initial parameters, when their minimum and maximum possible values are known, all functions, in particular, the objective function and the constraint functions, are also partially indefinite. Then the problem described above is reduced to minimizing the functions of the estimated costs determined at the minimum and maximum possible values of the initial partially indefinite parameters, as in [15]

$$
\begin{aligned}
& 3\left(a_{1}^{\min }, a_{2}^{\min }, \ldots, a_{m}^{\min }, x_{1}, x_{2}, \ldots, x_{n}\right) \rightarrow \min , \\
& 3\left(a_{1}^{\max }, a_{2}^{\max }, \ldots, a_{m}^{\max }, x_{1}, x_{2}, \ldots, x_{n}\right) \rightarrow \min
\end{aligned}
$$

taking into account the constraints (2) and functional constraints in the form of equality and inequality imposed on functions determined by the minimum and maximum possible values of the initial parameters

$$
\begin{aligned}
& f_{j}\left(a_{1}^{\min }, a_{2}^{\text {min }}, \ldots, a_{m}^{\text {min }}, x_{1}, x_{2}, \ldots, x_{n}\right)=\bar{f}_{j}^{\text {min }}, \quad j=\overline{1, N} \\
& f_{j}\left(a_{1}^{\text {max }}, a_{2}^{\max }, \ldots, a_{m}^{\text {max }}, x_{1}, x_{2}, \ldots, x_{n}\right)=\bar{f}_{j}^{\text {max }}, \quad j=\overline{1, N} \\
& g_{l}\left(a_{1}^{\min }, a_{2}^{\text {min }}, \ldots, a_{m}^{\text {min }}, x_{1}, x_{2}, \ldots, x_{n}\right) \leq \bar{g}_{l}^{\text {min }}, \quad l=\overline{1, L} \\
& g_{l}\left(a_{1}^{\max }, a_{2}^{\max }, \ldots, a_{m}^{\max }, x_{1}, x_{2}, \ldots, x_{n}\right) \leq \bar{g}_{l}^{\max }, \quad l=\overline{1, L}
\end{aligned}
$$

Solution of the obtained optimization problem (1a) - (4b) reduces to solving the equivalent two deterministic problems. In the first problem, the objective function is represented in the form (1a), and the constraints consist of (2), (3a) - (4b). In the second problem, the objective function form is (1b), and the constraints are (2), (3a) - (4b). As a result of solving these two deterministic problems, the interval values of the sought-for controlled parameters are determined. In this case, the lower boundary values of the intervals are obtained as a result of solving the first problem, and the upper boundary values are obtained as a result of solving the second problem. For the final solution of the original problem, one point is taken at which all the given constraints are satisfied. The coordinates of this point are determined by the values of the parameters found in the obtained intervals. The value of the objective function at the point of optimal solution is taken as an interval, the boundaries of which are determined by the results of solving the first and second deterministic problems.

\section{The results of calculation experiments}

To study the effectiveness of the described algorithm, we consider its application for solving the problem of power system mode optimization using the example of the test circuit (Fig. 1), given in [4] for given interval (partially undefined) values of active power of load nodes 2,3 , and 4 . Minimum and maximum possible values of loads in these nodes are given in table 1 . The active power of node 5 is deterministic and $P_{5}=500 \mathrm{MW}$.

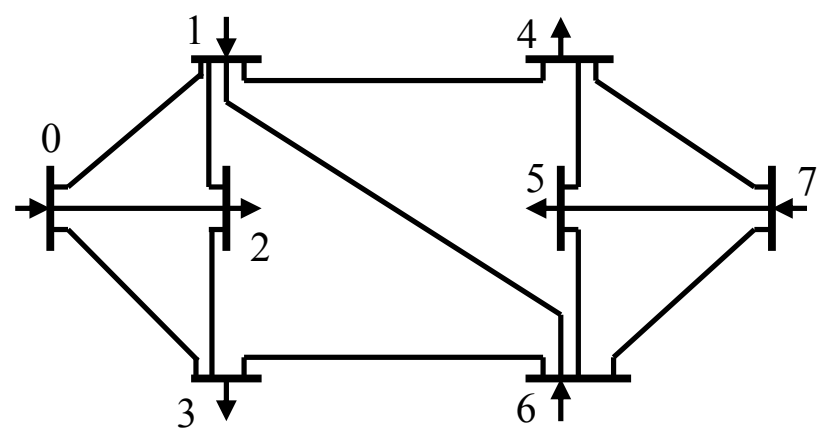

Fig. 1. The scheme of the power system.

Table 1. Minimum and maximum limit values of active loads.

\begin{tabular}{|c|c|c|}
\hline $\begin{array}{c}\text { The number of } \\
\text { node, } i\end{array}$ & $P_{i}^{\min }, \mathrm{MW}$ & $P_{i}^{\max }, \mathrm{MW}$ \\
\hline 2 & 380 & 420 \\
\hline 3 & 570 & 630 \\
\hline 4 & 190 & 210 \\
\hline
\end{tabular}

In four nodes $0,1,6$ and 7 there are thermal power plants (TPPs) with oil equivalent consumption characteristics, presented in the form

$$
B_{i}=c_{i}+d_{i} P_{i}+e_{i} P_{i}^{2}, \quad \text { t.o.e. } / \text { h. }
$$

where t.o.e./h.- tonne of oil equivalent per hour.

The values of coefficients of oil equivalent consumption characteristics of thermal power plants are given in table 2 . The minimum and maximum permissible capacities of all the TPPs are the same and, MW

$$
100 \mathrm{MBT}<P_{i}<1000 \mathrm{MBT}, \quad i=0,1,6,7 .
$$

In three power transmission lines (TPLs) 6-3, 6-5 and 0-3 the active power flow are controlled, MW:

$$
P_{6-3} \leq 480, \quad P_{6-5} \leq 70, \quad P_{0-3} \leq 100 .
$$

Power flows through a controlled TPLs are determined by linearized formulas using the node power distribution coefficients:

$$
P_{l}=\sum_{i \in \Gamma} C_{l i}^{\Gamma} P_{i}^{\Gamma}-\sum_{j \in H} C_{l j}^{H} P_{j}^{H}+P_{l 0}
$$


Table 2. The coefficients of oil equivalent consumption characteristics of TPPs

\begin{tabular}{|c|c|c|c|}
\hline $\begin{array}{c}\text { The node number } \\
\text { with TPP }\end{array}$ & $c_{i}$ & $d_{i}$ & $e_{i}$ \\
\hline 0 & 35 & 0,14 & 0,00035 \\
\hline 1 & 42 & 0,14 & 0,00042 \\
\hline 6 & 21 & 0,105 & 0,00028 \\
\hline 7 & 28 & 0,175 & 0,000175 \\
\hline
\end{tabular}

Table 3. Power distribution coefficients of nodes. where $C_{l i}^{\Gamma}, C_{l j}^{H}$ are the power distribution coefficients of the $i$-th generating and $j$-th load nodes by PTL $l$ (table. 3 ); $P_{i}^{\Gamma}$, $P_{j}^{H}$ are active powers of the $i$-th generating and $j$-th load nodes: $P_{l 0}$ is a free member of the linearized expression of power flow over the PTL $l$.

\begin{tabular}{|c|c|c|c|c|c|c|c|}
\hline \multirow{2}{*}{ PTL } & \multicolumn{7}{|c|}{ Nodes } \\
\cline { 2 - 8 } & 1 & 2 & 3 & 4 & 5 & 6 & 7 \\
\hline $6-3$ & 0,2536 & $-0,0713$ & $-0,172$ & 0,4106 & 0,489 & 0,6343 & 0,4986 \\
\hline $6-5$ & $-0,0701$ & 0,02025 & 0,0484 & $-0,281$ & $-0,4223$ & 0,1029 & $-0,2986$ \\
\hline $0-3$ & $-0,14$ & $-0,1735$ & $-0,267$ & $-0,1744$ & $-0,1917$ & $-0,224$ & $-0,1939$ \\
\hline
\end{tabular}

Thus, in the problem under consideration, functional constraints in the form of both equality and inequality are linear. The right-hand sides of functional constraints in the form of equality and inequality at interval node loads are determined based on interval arithmetic operations. As a result, we have the following interval values for the righthand sides of the constraint functions in the form of equality on power balance and inequality on power flow in the controlled PTLs 6-3, 6-5, and 0-3, respectively:

$[1640 ; 1760], \quad[664,168 ; 685,592], \quad[-164,877 ;-155,543]$,

$$
[-273,554 ;-247,106] \text {. }
$$

In the problem under consideration, the constraint in the form of equality is a condition for the active power balance in power system, therefore, the left-hand sides of the constraints in the form (3a) and (3b) are the same. When translating the components associated with the interval loads of the nodes in constraints in the form of inequality on power flows in the controlled NPLs, the left-hand sides of the constraints (4a) and (4b) also become the same. In this case, in each deterministic problem, one constraint in the form of equality takes part. In the first problem, the righthand side of the constraint is equal to the lower boundary value, and in the second, to the upper boundary value of the total load of power system specified in the interval form.

Since the left-hand sides of the constraints in the form of inequality obtained from (4a) and (4b) are the same, one of these constraints is involved in both deterministic problems, the right-hand side of which is less than the second. In this case, the second constraint with a large right-hand side turns into a passive restriction.

Thus, the first deterministic optimization problem, for the considered example, according to the described algorithm has the following form:

The objective function

$$
\begin{aligned}
& B=B_{0}+B_{1}+B_{6}+B_{7}=126+0,14 P_{0}+0,00035 P_{0}^{2}+0,14 P_{1}+ \\
& +0,00042 P_{1}^{2}+0,105 P_{6}+0,00028 P_{6}^{2}+0,175 P_{7}+ \\
& +0,000175 P_{7}^{2} \rightarrow \min
\end{aligned}
$$

Constraints

$$
\begin{aligned}
& P_{0}+P_{1}+P_{6}+P_{7}=1640, \\
& 0,2536 P_{1}+0,6343 P_{6}+0,4986 P_{7} \leq 664,168, \\
& -0,0701 P_{1}+0,1029 P_{6}-0,2986 P_{7} \leq-164,877, \\
& -0,14 P_{1}-0,224 P_{6}-0,1939 P_{7} \leq-273,554, \\
& 100<P_{i}<1000, \quad i=0,1,6,7 .
\end{aligned}
$$

The second deterministic problem is represented as follows:

The objective function

$$
\begin{aligned}
& B=B_{0}+B_{1}+B_{6}+B_{7}=126+0,14 P_{0}+0,00035 P_{0}^{2}+0,14 P_{1}+ \\
& +0,00042 P_{1}^{2}+0,105 P_{6}+0,00028 P_{6}^{2}+0,175 P_{7}+ \\
& +0,000175 P_{7}^{2} \rightarrow \min
\end{aligned}
$$

Constraints

$$
\begin{aligned}
& P_{0}+P_{1}+P_{6}+P_{7}=1760, \\
& 0,2536 P_{1}+0,6343 P_{6}+0,4986 P_{7} \leq 664,168, \\
& -0,0701 P_{1}+0,1029 P_{6}-0,2986 P_{7} \leq-164,877, \\
& -0,14 P_{1}-0,224 P_{6}-0,1939 P_{7} \leq-273,554, \\
& 100<P_{i}<1000, \quad i=0,1,6,7 .
\end{aligned}
$$

It should be noted that the first and second deterministic problems, for the considered example, differ from each other only by the right-hand side of the constraint in the form of equality.

In table 4 the results of solving the first and second deterministic problems are shown.

According to the results given in table 4, we determine the interval values of the optimal powers of the settlement plants and the total of oil equivalent consumption:

$P_{0}=[127,720 ; 247,701] \mathrm{MW}, \quad P_{l}=[547,769 ; 547,715]$

MW $, \quad P_{6}=[326,838 ; 326,673] \mathrm{MW}, \quad P_{7}=[637,673$;

637,910] MW, $\mathrm{B}=[599,280 ; 631,857]$ t.o.e./h. 
Table 4. The Results of solving the deterministic optimization problems.

\begin{tabular}{|c|c|c|c|c|c|c|c|}
\hline \multicolumn{4}{|c|}{ TPPs load, MW } & \multicolumn{3}{|c|}{ Power flow on controlled TPPs, MW } & \multirow{2}{*}{$\begin{array}{l}\text { The total fuel equivalent } \\
\text { consumption, t.o.e./h. } \\
\text { B }\end{array}$} \\
\hline$P_{0}$ & $P_{1}$ & $P_{6}$ & $P_{7}$ & $P_{6-3}$ & $P_{6-5}$ & $P_{0-3}$ & \\
\hline \multicolumn{8}{|c|}{ The results of solving the first deterministic problem } \\
\hline 127,720 & 547,769 & 326,838 & 637,673 & 480,00 & 39,70 & 100,00 & 599,280 \\
\hline \multicolumn{8}{|c|}{ The results of solving the second deterministic problem } \\
\hline 247,701 & 547,715 & 326,673 & 637,910 & 480,00 & 39,62 & 100,00 & 631,857 \\
\hline
\end{tabular}

For the final solution of the problem, according to the above marked rules, we take any point whose coordinates are determined by the powers of the plants in the received intervals. In this case, the lower and upper boundary values of power plant power intervals at nodes 1,6 , and 7 were the same. And the power of the balancing plant can be taken as the average value in the obtained interval. Thus, we take the optimal power of plants as

$\mathrm{P}_{0}=187 \mathrm{MW}, \mathrm{P}_{1}=547,75 \mathrm{MW}, \mathrm{P}_{6}=326,75 \mathrm{MW}$, $\mathrm{P}_{7}=637,75 \mathrm{MW}$.

The minimum total oil equivalent consumption in power system has an interval value: $\mathrm{B}=[599,280 ; 631,857]$ t.o.e./h.

\section{Conclusion}

The problems of power electric system development and functioning optimization taking into account simple and functional constraints in the form of equality and inequality in conditions of partial (interval) uncertainty of initial parameters, can be effectively solved by the method of determinization.

In power system mode optimization on active power and presenting the expression of power flow over a controlled PTL using linear distribution coefficients, solving the problem described by the method of determinization is much easier by halving the number of functional constraints in the form of equality and inequality compared to the classical optimization algorithm in basis of determinization method.

\section{References}

1. Methods for power system mode optimizaion. V.M. Gornstein, B.P. Miroshnichenko, A.V. Ponomarev and others; Edited by V.M.Gornstein (Energy, Moscow, 1981).

2. Krumm L.A. Methods of optimization and control of electric power systems (Nauka, Novosibirsk, 1980).
3. Automation of dispatch control in the electric power systems / Edited by Yu.N. Rudenko and V.A. Semenov (Publishing MPEI, Moscow, 2000).

4. Fazylov H.F., Nasyrov T.Kh. Steady state mode of power systems and its optimization (Moliya, Tashkent, 1999).

5. Nasirov T.Kh., Gayibov T.Sh. Theoretical foundations of power system mode optimization (Fan va texnologiya, Tashkent, 2014).

6. Gayibov T.Sh., Reimov K.M., Proc. of the Seventh All-Russian Scientific and Technical Conference with international participation. Energy: Management, quality and energy efficiency. Blagoveshchensk, (2013).

7. Gayibov T.Sh, Latipov Sh.Sh. Taking into Account of Functional Constraints in Optimization of Modes of Power Systems by Genetic Algorithms. Engineering, 11 (4), (2019).

8. Articles written on the occasion of the 50th anniversary of fuzzy set theory // Didier Dubois, Henri Prade. Rapport Interne IRIT RR-215-11-FR. December (2015).

9. M. Valdma, M. Keel, H. Tammoja, J. Shuvalova. Oil Shale, vol. 24, 2, Special, (2007).

10. Chaoyi Peng, Shunbo Lei, Yunhe Hou, Felix Wu. CSEE Journal of Power and Energy Systems, vol. 1, March 1, (2015).

11. Valdma M, Keel M, Liik O, Tammoja H. Proc. of IEEE Bologna PowerTech, Bologna, Italy, June 23-26, Bologna, Italy. Paper 252, (2003),

12. Arzamastsev D.A. and other. ACS and optimization of power system modes: (Higher. school, Moscow, 1983).

13. Gayibov T.Sh., Reimov K.M. Problems of energy and resource saving (special issue). - Tashkent -4, (2013).

14. V.I. Levin. Automatic Control and Computer Sciences, Vol. 46, 4, (2012).

15. V.I. Levin. XII All-Russian Meeting on Management Problems of the VSPU-2014. June 6-19, (2014).

16. Nemkova EA. Abstract of diss. for Degrees Ph.D., Penza, (2014). 